\title{
FINDING BEHAVIORAL MOTIFS IN FLY TRAJECTORIES*
}

\author{
DHRUV GROVER ${ }^{\dagger}$ AND SIMON TAVARÉ $\ddagger$
}

\begin{abstract}
Patterns of locomotor activity of a freely moving organism can help characterize its behavioral phenotypes. To infer behavior from such activity in Drosophila melanogaster, we use a real-time image acquisition system to track the movement of multiple flies in three dimensions. When dealing with fly movement trajectories, we must take into account that similar movement patterns can be expressed in different orientations and speeds. In this paper, we present methods to transform the three-dimensional fly movement trajectories into a space that is translation, rotation, scale and timescale invariant. We then propose an approach motivated by sequence alignment to detect similar movement patterns from fly trajectories in order to infer specific behaviors. We demonstrate the accuracy of the methods and highlight their usefulness in studies aimed at characterizing behavioral phenotypes.
\end{abstract}

1. Introduction. The holy grail of biology today is understanding how the genotype of an organism influences phenotypes such as behavioral characteristics. One way to study behavior in animals is to model and analyze their movement as time-series trajectories. In this paper, we develop methods to extract behaviors of interest from movement of the fruit fly, the model organism Drosophila melanogaster. Our approach is motivated in part by sequence alignment methods, a perfect topic for this dedicatory issue honoring Michael Waterman's numerous important contributions to computational molecular biology.

The apparent simplicity of identifying and characterizing courtship behavior in Drosophila melanogaster has made it one of the most popular model organisms for behavioral genetics. A male fly isolated since birth is capable of displaying the full repertoire of sequence-dependent patterns in the courtship ritual when it encounters a female, leading to the notion that this behavior is genetically programmed [2]. Because of their apparent lack of flexibility, such behaviors are termed "fixed action behaviors" (see Figure 1.1). This gives us a unique opportunity to characterize male courtship behavior using its trajectory as it tries to mate with the female.

\section{Methods.}

2.1. Setup. Flies were placed in standard $25 \times 75 \mathrm{~mm}$ polyethylene culture vials with food at the bottom and stoppered with cotton at the top. The vial was placed in the center of a circular camera rig, $70 \mathrm{~cm}$ in diameter. Six calibrated and synchronized

\footnotetext{
*Dedicated to Michael Waterman on the occasion of his 67th birthday.

${ }^{\dagger}$ Molecular and Computational Biology, University of Southern California, Los Angeles, California 90089-2910. Current address: Howard Hughes Medical Institute, Janelia Farm Research Campus, 19700 Helix Drive, Ashburn, Virginia 20147. E-mail: groverd@janelia.hhmi.org

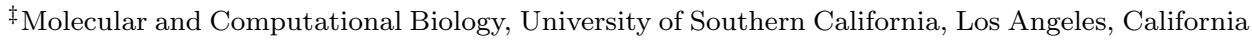
90089-2910. E-mail: stavare@usc.edu
} 


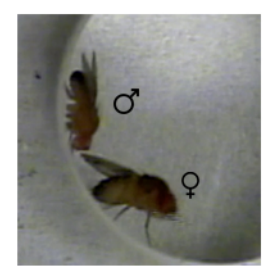

Following

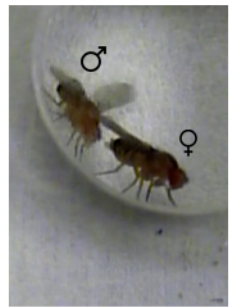

Wing Vibration

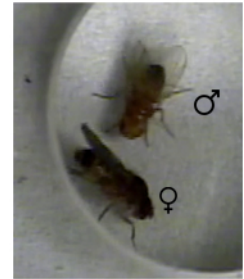

Orientation

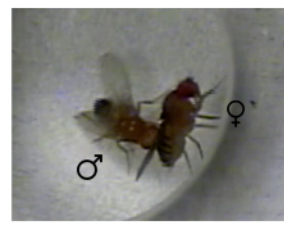

Licking

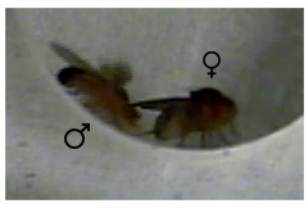

Tapping

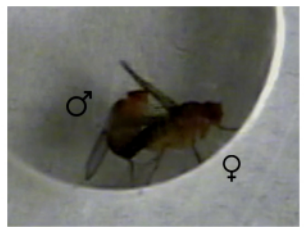

Attempted Copulation

FIG. 1.1. Sequence of genetically programmed fixed-action patterns performed by the Drosophila male exhibiting courtship behavior. The sequence of events moves along the top row, then along the bottom row.

Flea digital cameras (Point Grey) were mounted on the camera rig, facing downward at a distance of $15 \mathrm{~cm}$ from the vial. Each camera was fitted with an $8 \mathrm{~mm}$ megapixel fixed focus lens (Edmund Optics). See Figure 2.1. To track flies in the dark, the camera setup was modified with a red-wavelength light source, since flies cannot perceive red light. For courtship tracking assays, single male and female flies were placed in a vial together.

2.2. Tracking. Detection and tracking of flies in three-dimensional space was accomplished using the real-time image acquisition system mentioned above and algorithms described in our previous paper [3]. The system uses an array of calibrated and synchronized cameras to detect multiple flies and integrates the detected fly silhouettes to construct three-dimensional visual hulls of each fly. An extended Kalman filter is then used to track each fly in three-dimensional space, i.e., estimate its state from the reconstructed visual hulls, given past positions.

2.3. 3D Trajectory Transformation. Let $\boldsymbol{P}$ be a time-series of three-dimensional vectors describing the position of a fly at time instants $0,1, \ldots, n-1$ :

$$
\boldsymbol{P}=\left[P_{0}, \ldots, P_{n-1}\right]=\left[\left(x_{0}, y_{0}, z_{0}\right), \ldots,\left(x_{n-1}, y_{n-1}, z_{n-1}\right)\right]
$$

Designing distance measures invariant to rotation, translation and scale using only the three-dimensional spatial information is a challenging task. Instead, we use a modification of the technique from [9] to transform three-dimensional fly trajectories 


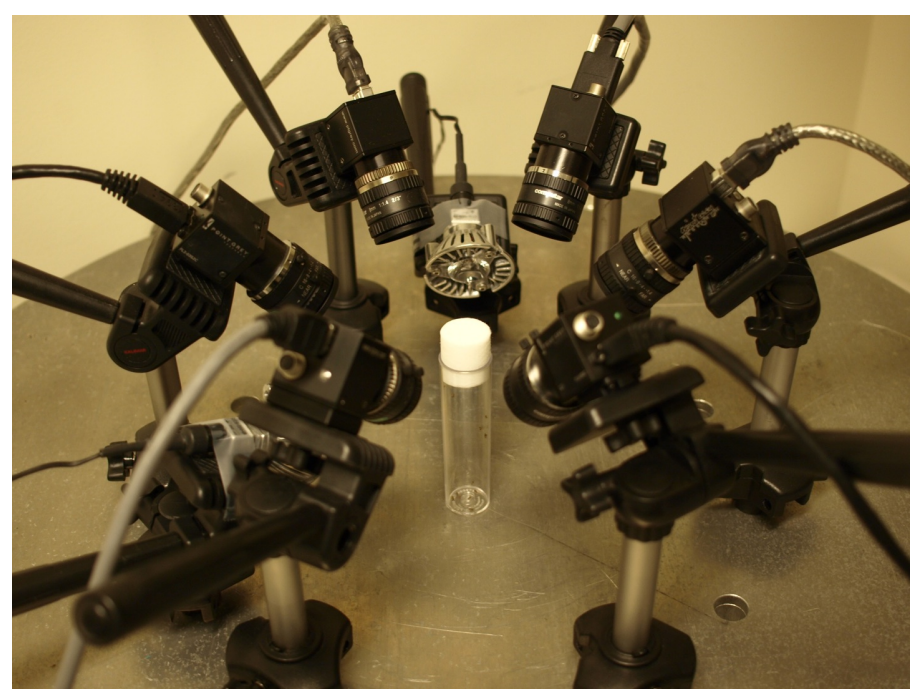

FIG. 2.1. Experimental setup of six cameras on the circular rig. Flies are placed in the vial in the center.

to a representation more amenable to this purpose.

The first step in designing such a distance measure is to determine the movement vector $\boldsymbol{M}$ from the positional information at time $t$, using differences:

$$
M_{t}=P_{t}-P_{t-1}, \quad t=1, \ldots, n-1 .
$$

The angle between each movement vector $M_{t}$ and a reference movement vector $M_{\text {ref }}$ is determined next. We use the positive $\mathrm{x}$-axis, the vector $(1,0,0)$, as a reference vector for our experimentation. Different approaches to choosing reference vectors are outlined in [9].

The angle $\alpha_{t}$ required to align the vector $M_{\text {ref }}$ to the current movement vector $M_{t}$ is measured by the smallest angle between the two three-dimensional vectors, which lies between 0 and $\pi$ radians. It is computed by

$$
\alpha_{t}=\tan ^{-1}\left(\frac{\left\|M_{t} \times M_{\mathrm{ref}}\right\|}{M_{t} \cdot M_{\mathrm{ref}}}\right), \quad t=1, \ldots, n-1,
$$

where $A \times B$ refers to the cross product and $A \cdot B$ refers to the scalar product of the vectors $A$ and $B$.

This angle can also be measured using the cosine formula specified in [9], but that is subject to increasing errors as the angle approaches $\pm \pi$, i.e., when the two vectors $M_{t}$ and $M_{\text {ref }}$ are nearly parallel. To avoid such a scenario we use (1), which combines the scalar product of the two vectors with the vector cross product in order to use both the sine and cosine in calculating the angle accurately.

In addition to the angle between each movement vector and the reference vector, we also determine the length of the movement vector (arc-length). We have there- 
fore transformed the trajectory from a time-series of three-dimensional vectors into a sequence of (angle, arc-length) pairs. This transformation will be referred to as the AAL representation of a three-dimensional trajectory;

$$
P_{\mathrm{AAL}}=\left[\left(\alpha_{1},\left\|M_{1}\right\|\right), \ldots,\left(\alpha_{n-1},\left\|M_{n-1}\right\|\right)\right]
$$

The goal of transforming trajectories in three-dimensional spatial coordinates to the AAL representation is to achieve rotation, translation and scale invariance. In the transformation described above, rotating a trajectory will result in a vertical shift in the AAL space (see Figure 2.2(b)). To achieve rotational invariance we normalize the AAL representations by iteratively subtracting the average angle value [9]. Figures 2.2(a), 2.2(b) and 2.2(c) demonstrate this technique. In Figure 2.2(a) the red trajectory is identical to the blue trajectory, except for a rotation of 90 degrees about the Z-axis. When these trajectories are transformed into their respective AAL representations (Figure 2.2(b)), they appear vertically separated by roughly 1.5 radians which corresponds to the 90 degree rotation. The AAL representations match exactly after normalization, demonstrating the rotation and translation invariance property of this transformation (Figure 2.2(c)). If scale invariance is desired, it can be achieved by dividing the length of each movement vector by the total trajectory length.

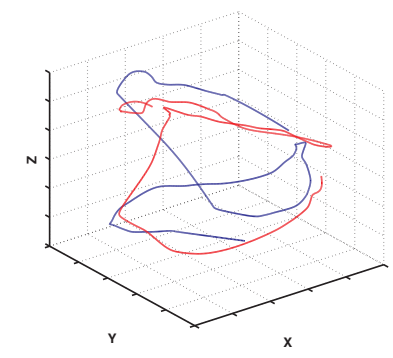

(a)

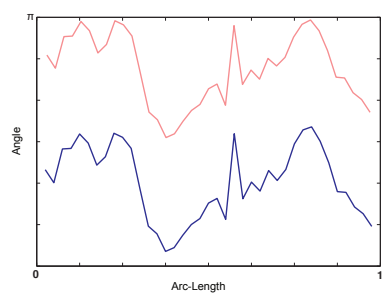

(b)

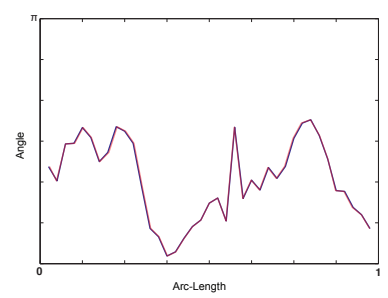

(c)

FIG. 2.2. (a) Two three-dimensional trajectories, (b) transformed to AAL representation without normalization, (c) with normalization. The red trajectory is identical to the blue trajectory except for a 90 degree rotation about the Z-axis, demonstrating rotation and translation invariance.

2.4. Sampling points by spline interpolation. In the previous section, we described a transformation to AAL space to achieve translation, rotation and scale invariance in three-dimensional fly trajectories. Another aim of our transformation process is to achieve time-scale invariance, because some flies might take longer than others to exhibit the same behavior. Therefore, their paths might appear similar but are traversed at different speeds. We demonstrate this scenario in Figure 2.3. In this figure, the red trajectory is identical to the blue trajectory except the fly traversed the same path at half the speed for the first half of the trajectory and twice the 
speed for the second half. When these trajectories are transformed into their AAL representations they match, demonstrating the time-scale invariance property of this transformation.

In the next section, we describe a bioinformatics-inspired Fitting Alignment (FA) approach that matches two trajectories by scoring their similarity. In order to make use of the one-dimensional FA, we assume that the measurements (angles) are taken at discrete and equi-spaced intervals (arc-lengths). In the example of Figure 2.3(b), even though the two trajectories match each other visually, the points are not equispaced on the arc-length axis: the red trajectory has twice as many (angle, arc-length) pairs as the blue trajectory for the first half of the path and half as many (angle, arclength) pairs for the second half. Without adjustments, these two trajectories would undoubtedly have a low similarity score even though they appear visually identical.

We therefore propose an approach that samples angle points in AAL sequences equi-spaced from each other on the arc-length axis to achieve a time-scale invariant transformation. We utilize splines to represent the sequences of (angle, arc-length) pairs, specifying the series of angle points at intervals along the curve and defining a function that allows additional points within those intervals to be calculated. We fit Catmull-Rom splines [1] to both AAL trajectory representations using the sequence of (angle, arc-length) pairs as control points. Once the splines are fitted to the AAL curves, we can sample equi-spaced points on them.

There are many ways of choosing the sampling interval size. One option is to sample additional points on the faster of the two three-dimensional trajectories, thereby increasing the number of points in its AAL representation. This is time-scale invariant relative to the slower trajectory (and this is the approach we take in this paper). The speed of the fly can be determined by traversing its series of arc-length values. Another possibility would be to remove points from the slower of the two trajectories in order to match it to the faster trajectory. Once the FA algorithm returns a set of matches of the training pattern in the test pattern, we can calculate the time taken for the two matched paths to be traversed using their original AAL sequence arc-length values to judge their similarity.

2.5. Fitting Alignment. The FA is a variant of the sequence alignment approach [11] that finds the best fit of a short sequence in a longer one. The intuition behind using this algorithm is to locate trajectory patterns of the male courtship behavior within long time-series of fly movement trajectories. We apply the transformations to three-dimensional trajectories mentioned in the previous two sections to create rotation, translation, scale and time-scale invariant representations which are then used for the alignment process.

We consider the sequence of equi-spaced angle points derived from two threedimensional trajectories in AAL time-scale invariant representation, $\boldsymbol{A}=A_{1} A_{2} \ldots A_{n}$ 


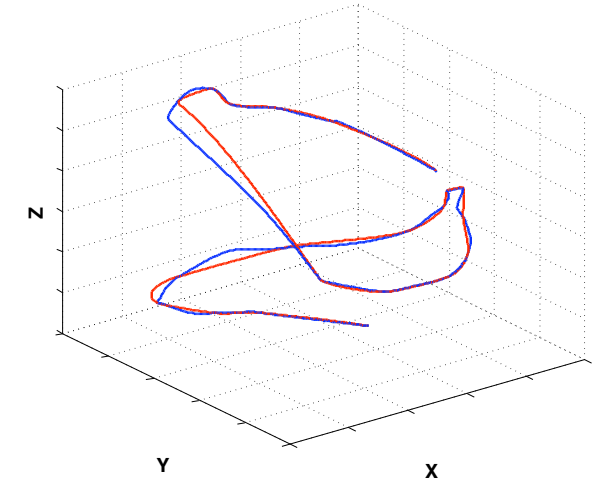

(a)

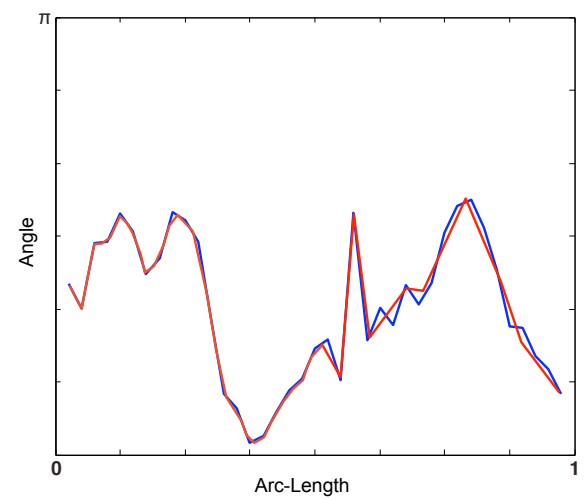

(b)

FIG. 2.3. Two three-dimensional trajectories, (a) transformed to AAL representation, (b) demonstrating time-scale invariance. The red trajectory is identical to the blue trajectory except it is half the speed for the first half of the path and twice the speed for the second half.

and $\boldsymbol{B}=B_{1} B_{2} \ldots B_{m}$ where $n<<m$. For a similarity measure $s$, our problem is to find

$$
T(A, B)=\max \left\{s\left(A, B_{k} B_{k+1} \ldots B_{l}\right), \quad 1 \leq k \leq l \leq m\right\}
$$

The assumption that sequence $\boldsymbol{A}$ is much shorter than sequence $\boldsymbol{B}$ is a valid one in our case because we are searching for patterns of fly behavior usually one to two minutes in length in long movement trajectories possibly on the order of hours or days.

Like other similarity based alignment methods, FA uses negative similarity to terminate poor matches which cannot be replicated in alignment methods based on distances between patterns. We modify the original one-dimensional FA algorithm using techniques outlined in $[6,7]$ to incorporate a distance-based matching function, a matching threshold and a non-matching penalty transforming the similarity based method to match patterns within equi-spaced angle sequences derived from the AAL representations of three-dimensional trajectories.

When the Euclidean distance between points $A_{i}$ and $B_{j}$ is less than a matching threshold of $\theta$ radians, denoting a matching state, we assign a positive score of $\alpha$. A non-matching state arises when the Euclidean distance is equal to or larger than the matching threshold $\theta$, in which case we assign a penalty of $-d\left(A_{i}, B_{j}\right)$. In the case of indels (insertions/deletions) a linear gap model with a gap penalty $\delta$ is adopted. Details about the values used for these alignment parameters are given in Section 3.1.

To find the fit for AAL trajectory pattern $\boldsymbol{A}$ within $\boldsymbol{B}$ using the proposed FA, the following recursion is applied to the dynamic programming matrix $T$ and the best 
match score is calculated. $T$ is initialised as

$$
T(i, 0)=-i \delta, \quad 0 \leq i \leq n ; \quad T(0, j)=0, \quad 0 \leq j \leq m
$$

and then

$$
T(i, j)=\max \left\{T(i-1, j-1)+s\left(A_{i}, B_{j}\right), T(i-1, j)-\delta, T(i, j-1)-\delta\right\}
$$

where

$$
s\left(A_{i}, B_{j}\right)= \begin{cases}\alpha, & \text { if } d\left(A_{i}, B_{j}\right)<\theta \\ -d\left(A_{i}, B_{j}\right), & \text { if } d\left(A_{i}, B_{j}\right) \geq \theta\end{cases}
$$

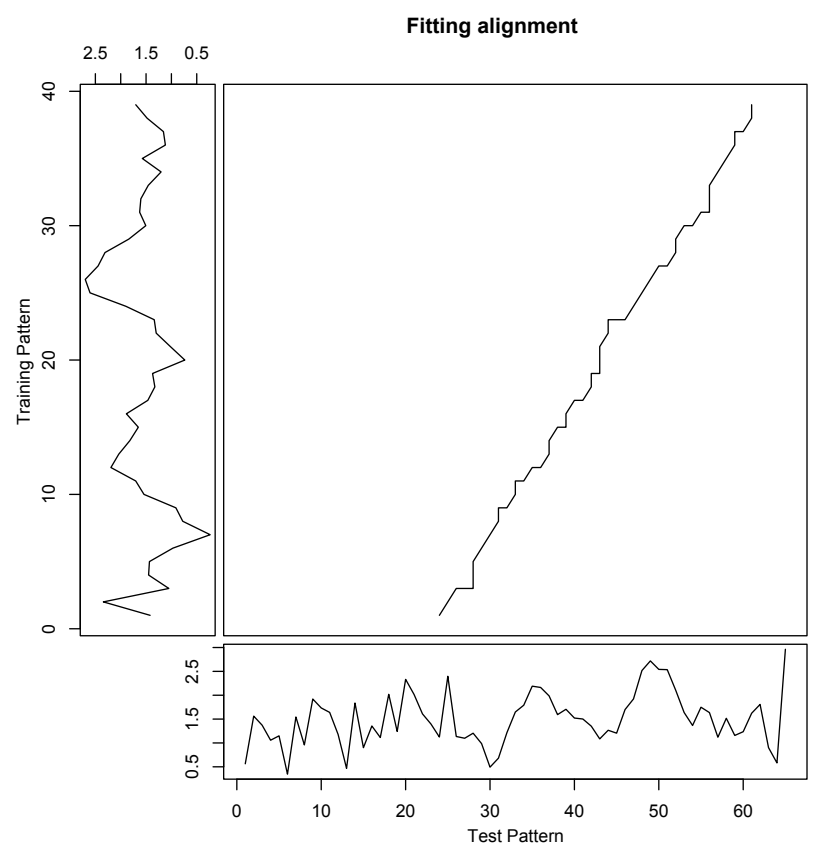

FIG. 2.4. Example extracting a matched segment of the training pattern from the test pattern from the Fitting Alignment matrix. The solid black line indicates the backtrace which gives us the alignment.

See Figure 2.4 for a demonstration of the FA approach in finding a match of the training pattern on the left embedded in a test pattern at the bottom of the figure. The solid black line represents the backtrace of similarity scores which gives us the aligned segments.

\section{Results.}


3.1. Fitting Alignment Parameters. Since our recognition task requires the patterns of behavior to be strictly matched to those used in the test set, we ideally want the matching threshold $\theta$ to be as small as possible. Some consideration is however required when choosing an appropriate matching threshold. Since similarity is derived from a distance-based function scoring the Euclidean distance between the two points in the AAL sequences being matched (see Section 2.4), if the selected $\theta$ is too large, dissimilar sequences might be construed to be similar, making the matching too general. On the other hand if $\theta$ is too small, similar sequences might not be recognized, making the matching too specific. The value of $\theta$ will therefore differ for different matching problems and possibly even for recognizing different behaviors. For our experiments in recognizing courtship behavior, we searched for the optimum value of $\theta$ in an interval of 0.1 to 0.5 radians in increments of 0.05 . For each increment of $\theta$, we found the optimum values for $\alpha$ and $\delta$ by cross-validating a set of accurately classified courtship sequences (15 training and 15 test). For the given dataset, $\theta=$ $0.25, \alpha=1.0$ and $\delta=2.0$ provided the best classification accuracy. We used these parameter values for testing the courtship recognition accuracy using our proposed alignment approach.

3.2. Recognizing Courtship Behavior. In order to test whether specific movement patterns are indicative of courtship behavior in flies, we performed the following test. We recorded 25 video sequences of the male fly exhibiting the courtship ritual and generated the 3D trajectories for them. Sequences of successful male courtship resulting in mating are usually in the range of 1-2 minutes in length. We then recorded 50 1-hour long video sequences, 25 of which are known to have one or more instances of the male fly exhibiting courtship and 25 without any courtship activity. We then generated the $3 \mathrm{D}$ trajectories for these 50 video sequences. The courtship training trajectories were extracted from movements of different male flies from those in the test trajectories to eliminate any bias in recognition accuracy. All 3D trajectories used in this test were transformed into AAL representation using the techniques mentioned in the previous section.

Next, we performed pairwise fitting alignments of the 25 training trajectories with each of the 50 test trajectories and determined the best alignment score for each pairing. A histogram of the frequency of the scores from trajectories with courtship activity (Red) and no courtship activity (Blue) is shown in Figure 3.1(a).

In Figure 3.1(b), we present a Receiver Operating Characteristic (ROC) curve for aligning the 25 training trajectories with the 50 test trajectories. We compare the operating characteristics of true positive rate (TPR) and false positive rate (FPR) for varying alignment scores. The curve is colorized according to the score cutoff values as indicated on the left. In Figure 3.1(c), we show the alignment accuracy values for different score cutoffs. 
The results in Figure 3.1 were based on using 25 courtship training sequences for the matching process. Next, we determined the effects of varying number of courtship training sequences on recognition accuracy. There are many combinations of choosing the training trajectories for sample sizes less than 25. For each iterate of a training sequence sample of size $n$ less than 25 , we choose 25 combinations of $n$ training trajectories from our pool of 25 trajectories without repetition. We then calculated the best alignment accuracy for each of the $25 n$ trajectory combinations and repeat this process for each sample size less than 25 . For the sample size 25, the alignment accuracy was determined earlier to be $90.6 \%$ (See Figure 3.1). In Figure 3.2 , we present the mean alignment accuracy results (with standard errors) by varying the number of courtship training sequences from 1 to 25 .

4. Discussion. In this paper we present an algorithm for measuring the similarity between three-dimensional trajectories and apply it to extracting path segments corresponding to the Drosophila male courtship ritual. We demonstrate that trajectories similar to those in the training set can be accurately identified and that these matched paths are representative of male fly courtship. This method can be generalized in several directions. The first is to utilize the topology of the space in which the flies are housed to determine a canonical coordinate system that is defined in terms of the fly trajectory data. Transforming the fly trajectories to such a coordinate system before conversion into AAL space would make the tasks of alignment and behavior identification more robust. In our experiments, flies are enclosed in cylindrical vials and it would be useful to transform the fly trajectories from a cartesian representation into a cylindrical coordinate system before applying the transformation to AAL space.

Flies are interacting insects, exhibiting myriad social behaviors. It would be interesting to see if the methods presented in this paper could be generalized to the vast array of social behaviors exhibited by the fruit fly. In fact, trajectories can be used to study complex behavior patterns, such as migration, foraging, territorial aggression and mating, in many animals. Extending our approach to study behavior in animals other than Drosophila could be a useful biological tool, especially in the fields of behavioral genetics, neuroscience, and ecology.

The Fitting Alignment belongs to a general class of dynamic programming algorithms. Instead of finding the best fit for a short trajectory in a long trajectory, the problem can be generalized to finding similar segments within two trajectories. The Smith-Waterman local alignment approach [8] would be the ideal approach to use for finding such matches. Since the alignment approach presented in this paper is one-dimensional, we transform the sequence of (angle, arc-length) pairs so that the measurements (angles) are at discrete and equi-spaced intervals (arc-lengths). A discussion about modifying the Smith-Waterman alignment approach to create a two- 


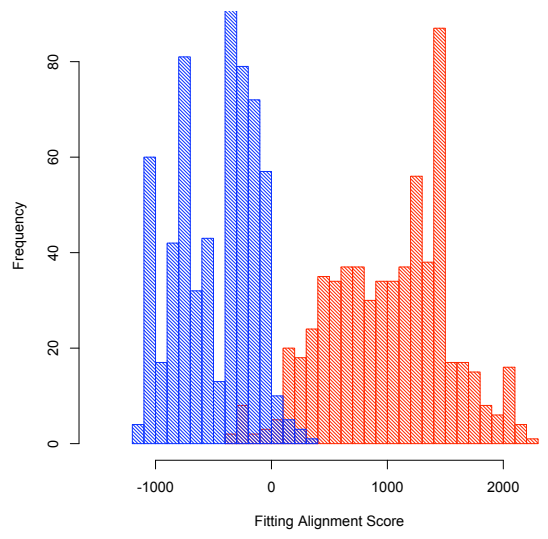

(a)

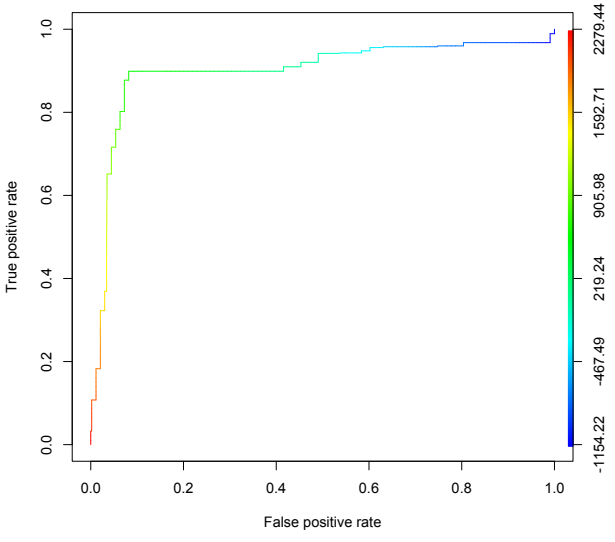

(b)

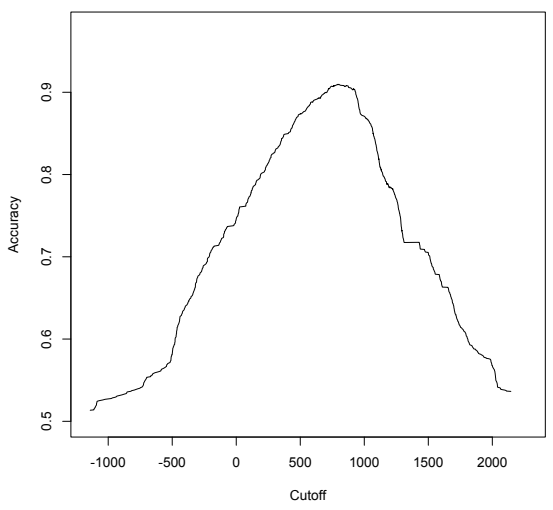

(c)

FIG. 3.1. (a) Histogram of pairwise alignment scores of fitting 25 courtship trajectories into 25 test trajectories containing one or more courtship events (Red) and 25 test trajectories containing no courtship events (Blue). ROC curve (b) and accuracy plot (c) of the data presented in (a).

dimensional alignment algorithm that would utilize both angles and arc-lengths in the alignment process is provided in [10].

In our previous work, we extended the real-time tracking system by exploiting fluorescent reporters for simultaneous tracking of movement and tissue-specific gene expression $[4,5]$. Since DsRED and GFP are used extensively as reporters for transgene expression in Drosophila and other organisms, methods to correlate movement and behaviors with levels of, and changes in, gene expression in vivo should provide insight into regulatory pathways. 


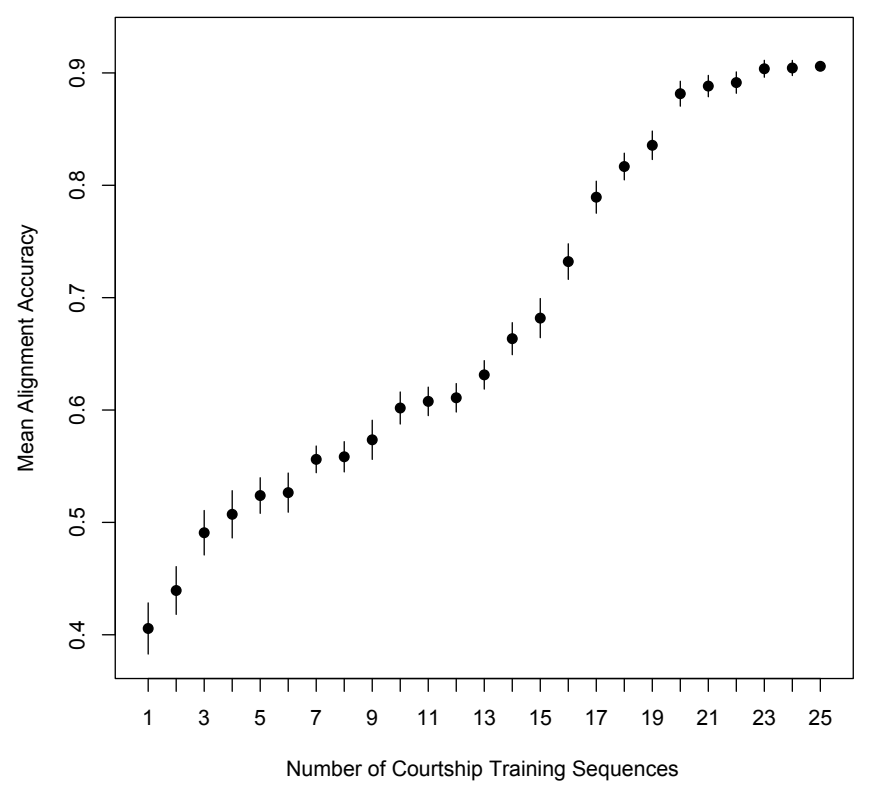

FIG. 3.2. Mean alignment accuracy with standard errors for varying number of courtship training sequences.

Acknowledgement. We thank Dr. Thomas D. Goldman for film of courtship behavior, Dr. Juan Nunez-Iglesias for the photo in Figure 2.1, and Dr. John Tower for comments on an earlier draft of the paper.

\section{REFERENCES}

[1] E. Catmull and R. Rom. A class of local interpolating splines. In: R. E. Barnhill and R. F. Riesenfeld, editors, Computer Aided Geometric Design, pages 317-326. Academic Press, New York, NY, USA, 1974.

[2] R. J. Greenspan and J. F. Ferveur. Courtship in Drosophila. Annu Rev Genet., 34(2000), pp. 205-232.

[3] D. Grover, J. Tower, and S. Tavaré. O fly, where art thou? Journal of the Royal Society Interface, 5(2008), pp. 1181-1191.

[4] D. Grover, J. Yang, D. Ford, S. Tavaré, And J. Tower. Simultaneous tracking of movement and gene expression in multiple Drosophila melanogaster flies using GFP and DsRED fluorescent reporter transgenes. BMC Research Notes, 2(2009), pp. 58.

[5] D. Grover, J. Yang, S. TAvaré, And J. Tower. Simultaneous tracking of fly movement and gene expression using GFP. BMC Biotechnology, 8(2008), pp. 93.

[6] D. Riedel, S. Venkatesh, And W. Liu. A Smith-Waterman local alignment approach for spatial activity recognition. Proceedings of the IEEE International Conference on Video and Signal Based Surveillance, 2006.

[7] D. Riedel, S. Venkatesh, And W. Liu. Recognising online spatial activities using a bioinfor- 
matics inspired sequence alignment approach. Pattern Recognition, 41(2008), pp. 34813492 .

[8] T. F. Smith And M. S. Waterman. Identification of common molecular subsequences. Journal of Molecular Biology, 147(1981), pp. 195-197.

[9] M. Vlachos, D. Gunopulos, And G. Das. Rotation invariant distance measures for trajectories. Proceedings of the tenth ACM SIGKDD International Conference on Knowledge Discovery and Data Mining, 2004.

[10] M. S. Waterman. Dynamic programming algorithms for picture comparison. Advances in Applied Mathematics, 6(1985), pp. 129-134.

[11] M. S. Waterman. Introduction to Computational Biology: Maps, Sequences and Genomes. Chapman \& Hall, 1995. 